\title{
Lift characteristics of two tandem airfoils in the hydrodynamically self-excited wake of a heated cylinder
}

\section{array}

\author{
Xiangyu Zhai ${ }^{1}$, Bo Yin ${ }^{1}$, Nader Karimi ${ }^{2,3}$, Larry K.B. Li ${ }^{1}$, Yu Guan ${ }^{1 *}$, Ao Wen ${ }^{4 *}$ \\ ${ }^{1}$ Department of Mechanical and Aerospace Engineering, The Hong Kong University of \\ Science and Technology, Clear Water Bay, Hong Kong
}

${ }^{2}$ School of Engineering and Computer Science, Queen Mary University of London, Mile End, E1 4NS, United Kingdom

${ }^{3}$ James Watt School of Engineering, University of Glasgow, Glasgow, G12 8QQ, United Kingdom

${ }^{4}$ Science and Technology on Combustion, Internal Flow and Thermo-structure Laboratory, Northwestern Polytechnical University, Xi'an 710072, China

*Corresponding authors:

Yu Guan (yguanad@connect.ust.hk)

Ao Wen (aw@nwpu.edu.cn)

\begin{abstract}
In this numerical study, the effect of a globally unstable wake - generated by one or two rigid cylinders - on the lift characteristics of two tandem airfoils is investigated via two-dimensional scale-adaptive simulations at a Reynolds number of 10000, with and without cylinder heating. The two airfoils have NACA 0012 profiles and are at the same angle of attack $\left(\alpha=10^{\circ}\right)$. When the two airfoils are positioned behind a single cylinder, their lift characteristics depend strongly on the horizontal spacing between themselves and the cylinder. Both the fore and aft airfoils experience periodic limit-cycle oscillations in the lift coefficient $\left(C_{l}\right)$ at a common natural frequency. Heating the cylinder to a temperature above that of the free-stream leads to monotonic decreases in the oscillation frequency and amplitude of $C_{l}$ for both airfoils. When the single cylinder is replaced by two tandem cylinders, $C_{l}$ oscillates less periodically, with broadband components appearing in the power spectra and with complex structures appearing in the attractors in phase space. The quantitative lift characteristics depend strongly on the horizontal spacing between the upstream and downstream cylinders. This study offers fresh insight into the effect of self-excited wake modes on the lift characteristics of tandem airfoils.
\end{abstract}




\title{
Nomenclature
}

\author{
c Chord length of airfoil \\ $C_{f} \quad$ Skin friction coefficient \\ $C_{l} \quad$ Lift coefficient \\ $D \quad$ Diameter of cylinder \\ $f \quad$ Frequency \\ $f_{1} \quad$ Frequency of dominant flow instability \\ G $\quad$ Gap height \\ L Horizontal distance between two tandem cylinders \\ LE Leading edge of airfoil \\ PSD Power spectral density \\ Re Reynolds number \\ RMS Root mean square \\ $S \quad$ Stagger distance \\ SAS Scale-adaptive simulation \\ St Strouhal number \\ $\triangle t \quad$ Time step \\ $T \quad$ Temperature of cylinder surface \\ TE Trailing edge of airfoil \\ U Free-stream velocity \\ $U_{\tau} \quad$ Friction velocity \\ $X \quad$ Horizontal distance between cylinder and the fore airfoil \\ $y^{+} \quad$ Dimensionless wall distance \\ $\Delta y_{1} \quad$ First-cell height
}

\section{Greek symbols}
$\alpha \quad$ Angle of attack
$\rho \quad$ Density of fluid
$\mu \quad$ Dynamic viscosity
$\tau \quad$ Time delay in phase space reconstruction
$\tau_{\omega} \quad$ Wall shear stress 


\section{Introduction}

In a world where pollution standards are becoming increasingly stringent and where the threat of global warning continues to rise, it is important to have access to technologies capable of fostering sustainable energy and enhancing energy efficiency and independence. To this end, technologies for harvesting energy, enhancing heat transfer and increasing thermal efficiency have attracted growing interest from various fields [1-7]. In particular, the phenomenon of vortex-induced vibration (VIV) has been extensively studied for the purpose of harvesting the kinetic energy in a free-stream flow and converting it into mechanical energy, which can then be converted to electrical energy via various mechanisms [8]. For example, placing a freely-moving airfoil in the unsteady supercritical wake of a cylinder can facilitate the harvesting of kinetic energy from the free-stream flow. Compared with conventional rotary-turbine-based energy harvesters, such VIV-based energy harvesters tend to operate at higher efficiencies under certain conditions. Energy harvesting from a free-stream flow has previously been explored by McKinney and DeLaurier [9]. It was found that the efficiency of a fluttering airfoil can be up to $8 \%$ higher than that of a rotary-turbine-based energy harvester operating at low Reynolds numbers. Moreover, Lau et al. [10] carried out experiments in a wind tunnel and found that the oscillation frequency and amplitude of a moving NACA 0012 airfoil mounted in the unsteady wake of a cylinder are strongly dependent on the position of the airfoil, particularly the horizontal and vertical separation distances relative to the cylinder itself. Derakhshandeh et al. [11] investigated a similar cylinder-airfoil configuration in a free-stream flow and came to a similar conclusion.

The aerodynamics of a single airfoil in isolation has been studied extensively in the past, both through experimental testing in wind tunnels and through numerical simulations [12-19]. Moreover, the lift characteristics of a single airfoil under the influence of an unsteady cylinder wake has also been studied before. However, relatively little is known about the lift characteristics of two tandem airfoils [20] subjected to the unsteady wake of a cylinder, or that of a pair of cylinders arranged in a tandem configuration, i.e. tandem cylinders. This is especially true when the wake is hydrodynamically self-excited in the sense that it is globally unstable owing to the presence of a sufficiently large region of local absolute instability [21]. In contrast to a locally convectively unstable flow, which acts as a hydrodynamic amplifier, a globally unstable flow acts as a hydrodynamic oscillator. Such a flow, therefore, oscillates at its own natural (self-excited) frequency and is relatively unresponsive to perturbations applied externally at other frequencies [21]. Such global modes can arise not only in wake flows [22] but also in jet flows [23-31] and in some diffusion flames [32,33].

The aim of the present study is to use two-dimensional numerical simulations to investigate how the lift characteristics of two tandem airfoils are affected by the presence of a globally unstable wake produced by one or two cylinders. This paper is 
organized into four parts. In the first part (Section 3), we examine the globally unstable wake of a single cylinder in order to validate our numerical setup and to establish a reliable baseline for subsequent comparisons with tandem airfoils. In the second part (Section 4), we place two tandem airfoils at different positions in the globally unstable wake of a single cylinder, and then examine how the lift characteristics of the airfoils are affected by their horizontal positon relative to the cylinder. In the third part (Section 5), we replace the single cylinder with two tandem cylinders, but keep the two tandem airfoils as before. Keeping the horizontal distance between the downstream cylinder and the two tandem airfoils constant, we investigate the influence of the horizontal separation distance between the upstream and downstream cylinders in terms of the lift coefficient and its frequency and amplitude of oscillation. In the fourth part (Section 6), we revisit the cylinder-airfoil configuration from the second part in order to investigate how heating the cylinder body affects the lift characteristics of the two tandem airfoils.

\section{Numerical setup}

\subsection{Flow configuration and airfoil geometry}

A rigid stationary cylinder of diameter $D=0.03 \mathrm{~m}$ is set in a two-dimensional computational domain, where the boundary condition on the left semi-circle edge is defined to be a velocity inlet and that on the right edge is defined to be a pressure outlet, as shown in Fig. 1. To minimize blockage effects, a vertical spacing of $10 D$ is imposed both above and below the cylinder axis, while a horizontal spacing of $15 D$ is imposed in the downstream (streamwise) direction.

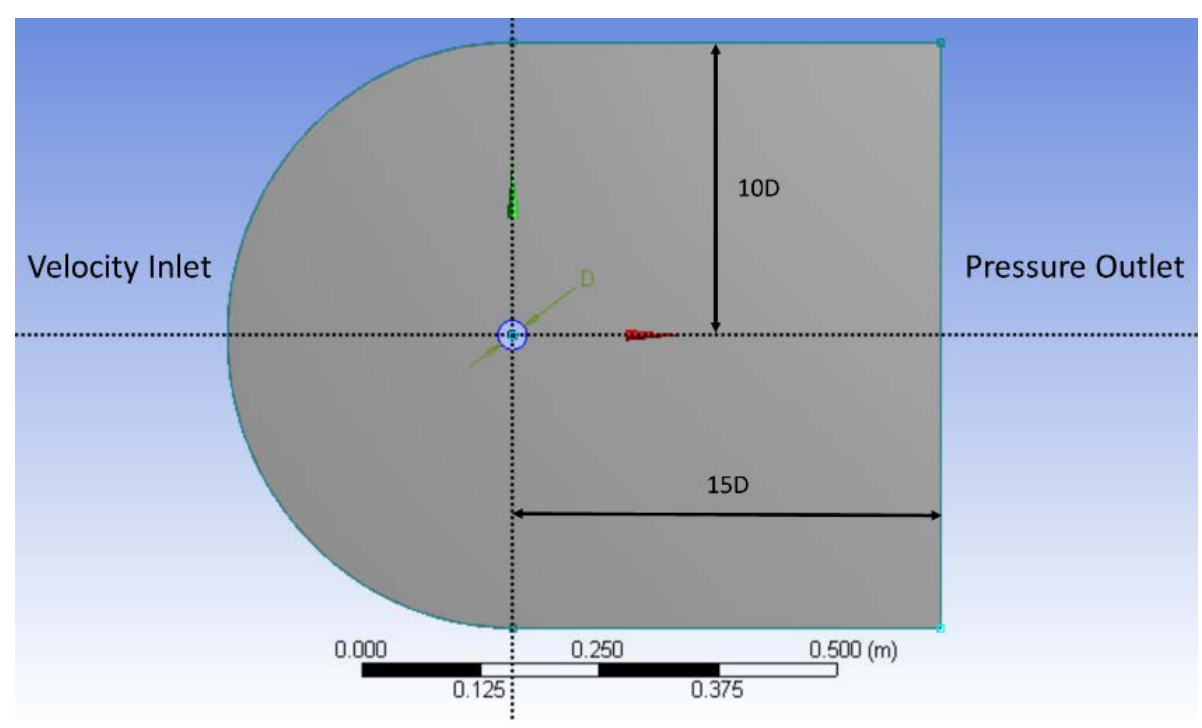

Figure 1: Geometry of the computational domain. 


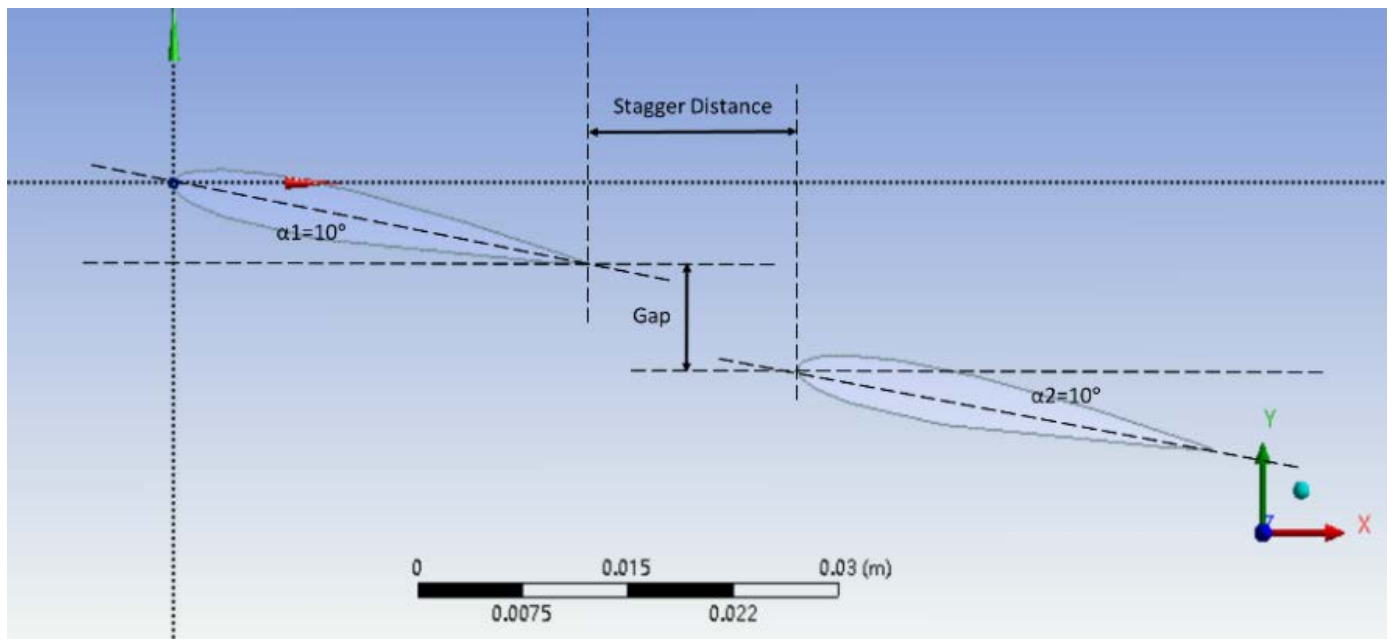

Figure 2: Geometry of the two tandem airfoils.

Fig. 2 shows the geometry of the two tandem airfoils considered in the present simulations. Both the fore airfoil and the aft airfoil have NACA 0012 profiles with a chord length of $c=0.03 \mathrm{~m}$. The stagger distance, which is defined as the horizontal distance between the trailing edge (TE) of the fore airfoil and the leading edge (LE) of the aft airfoil, is set to $S=0.5 c$. The gap height, which is defined as the vertical distance between the TE of the fore airfoil and the LE of the aft airfoil, is set to $G=0.4 c$. To minimize decalage effects, both tandem airfoils are oriented at an angle of attack of $\alpha=10^{\circ}$, which remains fixed throughout this entire study.

\subsection{Mesh generation}

An unstructured mesh consisting of quadrilateral elements is used, as shown in Fig. 3. Panel (a) is a magnified view of the airfoil, and panel (b) is a magnified view of the cylinder.

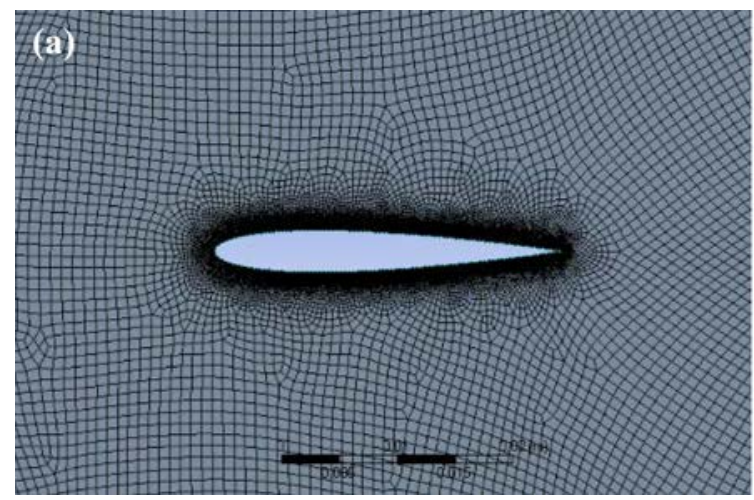

Figure 3: Unstructured mesh around (a) the airfoil and (b) the cylinder.

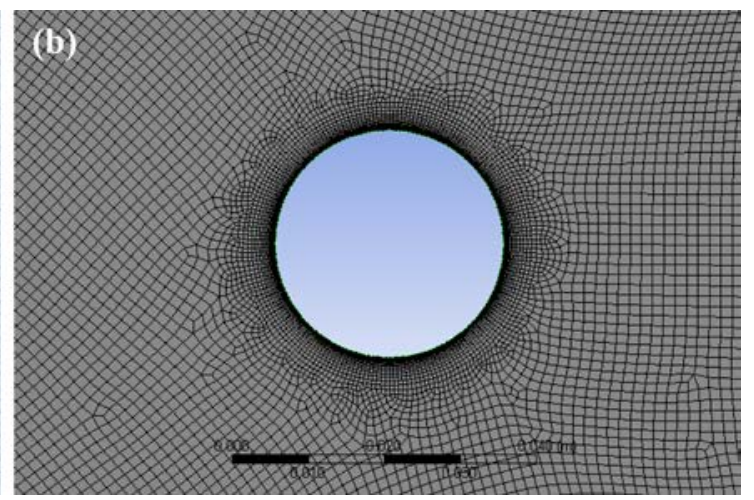

In airfoil simulations at the Reynolds numbers considered here $(R e=10000)$, it is critical that an appropriate dimensionless wall distance $y^{+}$be used to resolve the boundary layer based on a chosen turbulence model. For the turbulence model used here (to be discussed below), $y^{+}=1$ is an appropriate choice for the wall treatment, 
ensuring that around 10 to 15 grid layers are covered by the boundary layer [34]. The first-cell height $\Delta y_{1}$ can be determined as follows. Taking $R e=10000$, a density of $\rho=1.225 \mathrm{~kg} / \mathrm{m}^{3}$ (air), a dynamic viscosity of $\mu=1.7894 \times 10^{-5} \mathrm{~kg} /(\mathrm{m} \cdot \mathrm{s}$ ) and a cylinder diameter of $D=0.03 \mathrm{~m}$, we obtain a free-stream velocity of $U=4.87$ $\mathrm{m} / \mathrm{s}$. The dimensionless wall distance is defined as $y^{+}=\rho U_{\tau} \Delta y_{1} / \mu$, where $U_{\tau}=$ $\sqrt{\tau_{\omega} / \rho}$ is the friction velocity, $\tau_{\omega}=\frac{1}{2} C_{f} \rho U^{2}$ is the wall shear stress, and $C_{f}=$ $0.058 R e^{-0.2}$ is the skin friction coefficient. All the grid points near the cylinder and airfoils are refined such that the first-cell height is $5.0 \times 10^{-5} \mathrm{~m}$. A growth rate of 1.05 is applied to ensure that at least 10 grid layers are covered by the boundary layer.

\subsection{Numerical solver and turbulence model}

Low-Reynolds-number flows can pose challenges to the selection of an appropriate turbulence model. Derakhshandeh et al. [11] have found that the scale-adaptive simulation (SAS) turbulence model can perform well in the transient flow regime at $R e$ $=$ 10000. Another successful application of the SAS turbulence model was demonstrated by Maliska et al. [35] at $R e=11000$. This particular turbulence model uses the von Karman length scale, which enables it to adapt its behavior on the basis of the stability parameters of the flow at hand [36]. This also enables the SAS turbulence model to provide a robust balance between contributions from the simulated and resolved parts of the turbulence stresses. Consequently, the SAS turbulence model can automatically switch from large-eddy simulation to Reynolds-averaged-Navier-Stokes simulation. In the present study, we use the SAS turbulence model, with curvature correction activated for all the test cases. A pressure-based solver (ANSYS Fluent) is used to perform the numerical simulations, with a bounded second-order implicit transient scheme. The time step $\Delta t=0.00001 \mathrm{~s}$ is chosen to be small enough that the Courant-Friedrichs-Lewy number is less than unity throughout the domain, including near the surfaces of the cylinder and airfoils.

\subsection{Grid independence}

Grid independence is checked by varying the number of nodes in the central region of the computational domain around the cylinder, as shown in Fig. 4. 

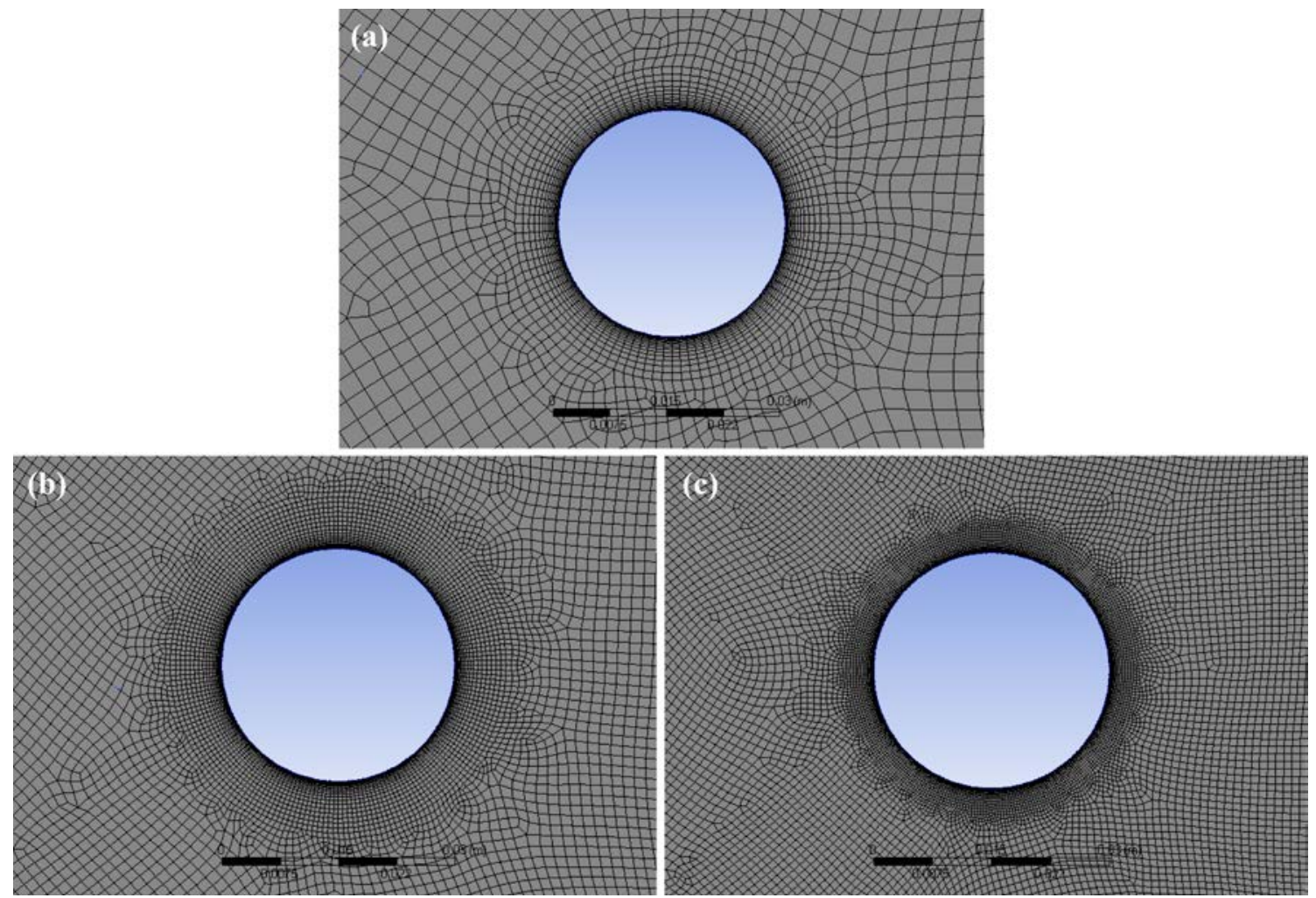

Figure 4: Mesh details around the cylinder with (a) 200, (b) 300 and (c) 400 nodes.

Magnified views of the mesh with 200, 300 and 400 nodes are shown in Fig. 4. The non-dimensional frequency of vortex shedding, which is known as the Strouhal number (St), is used to quantify the unsteady wake dynamics arising from the presence of a global hydrodynamic mode. Here the Strouhal number is defined as $S t \equiv f_{1} D / U$, where $f_{1}$ is the frequency of the dominant flow instability and is found by computing the power spectral density (PSD) from time traces of the lift coefficient. From Table 1, it can be seen that the coarsest mesh (with only 200 nodes) is already quite capable of resolving the unsteady wake dynamics. To be conservative, we use the 300-node mesh (Fig. 4(b)), as adding more nodes than this does not lead to further changes in St. In simulations of the flow around a single cylinder, 300 nodes around the cylinder correspond to a total of 35832 quadrilateral elements in the mesh.

\begin{tabular}{|c|c|}
\hline No. of nodes & St \\
\hline 200 (Fig. 4(a)) & 0.1976 \\
\hline 300 (Fig. 4(b)) & 0.2006 \\
\hline 400 (Fig. 4(c)) & 0.2006 \\
\hline
\end{tabular}

Table 1: Variation of the Strouhal number with the number of nodes in the mesh around the cylinder. 


\section{Numerical validation}

Before investigating the lift characteristics of two tandem airfoils under the influence of a self-excited cylinder wake, we first examine vortex shedding in the wake of a cylinder at $R e=10000$. Fig. 5 shows (a) a time trace of the lift coefficient of the cylinder, (b) its short-time PSD, and (c) its phase portrait and Poincaré map. The lift coefficient oscillates periodically in time at a well-defined natural frequency, as evidenced by the sinusoidal nature of the waveform and by the presence of a dominant peak in the PSD. This oscillatory behavior is characteristic of a von Karman vortex street, which is a nonlinear manifestation of a global hydrodynamic mode arising from a sufficiently large region of local absolute instability [21]. The phase portrait contains a closed periodic orbit, while the Poincaré map shows two discrete intercepts. These features point to the presence of a self-excited limit cycle arising from a Hopf bifurcation [21]. Such self-excited behavior arises not only in hydrodynamic systems such as bluff-body wakes [22], low-density jets [23-31] and jet diffusion flames [32,33], but also in various other flow systems, most notably those whose oscillations are self-excited by the positive feedback between (i) heat-release-rate oscillations from a flame or electric heater and (ii) the surrounding acoustic field [37-49].
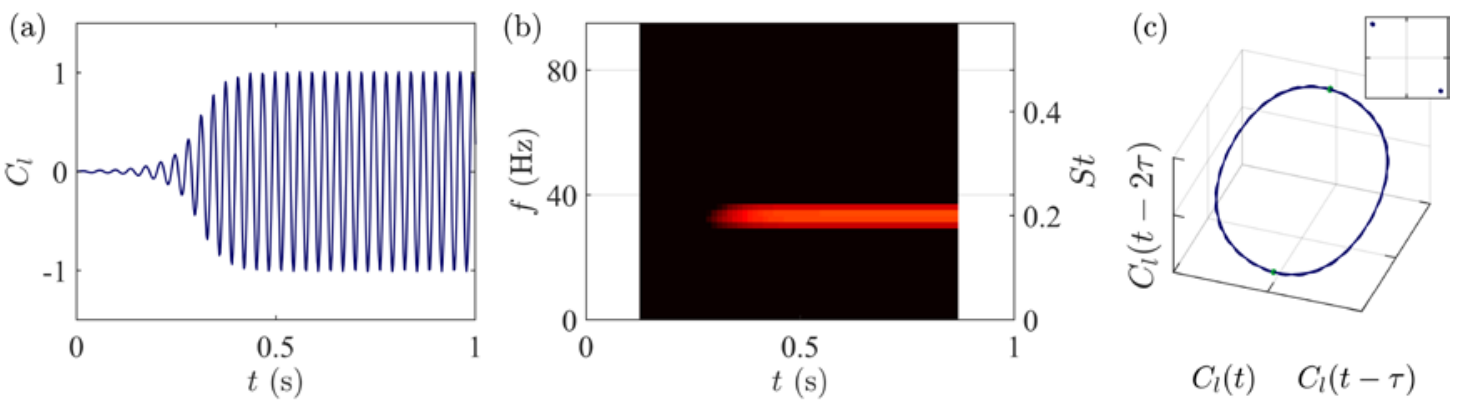

Figure 5: (a) Time trace, (b) PSD and (c) phase portrait and Poincaré map of the lift coefficient of a cylinder at $R e=10000$.

By examining the PSD shown in Fig. 5(b), we extract the Strouhal number of the dominant global mode as well as the corresponding oscillation amplitude, expressed here as the root mean square (RMS) amplitude of $C_{l}$. In Fig. 5(b), a sharp peak can be identified in the PSD at $S t=0.2006$, with an RMS amplitude of $C_{l}=0.5088$. These values for the global hydrodynamic mode agree well with both experimental and numerical data published in the literature, as the comparison in Table 2 shows.

\begin{tabular}{|c|c|c|}
\hline Source & St & RMS of $\boldsymbol{C}_{\boldsymbol{l}}$ \\
\hline Dong \& Karniadakis [50] (Direct numerical simulations) & 0.203 & 0.448 \\
\hline Gopalkrishnan [51] (experiments) & 0.193 & 0.384 \\
\hline
\end{tabular}




\begin{tabular}{|c|c|c|}
\hline Derakhshandeh et al. [11] (SAS) & 0.199 & 0.502 \\
\hline The present study (SAS) & 0.2006 & 0.5088 \\
\hline
\end{tabular}

Table 2: Numerical validation against published data.

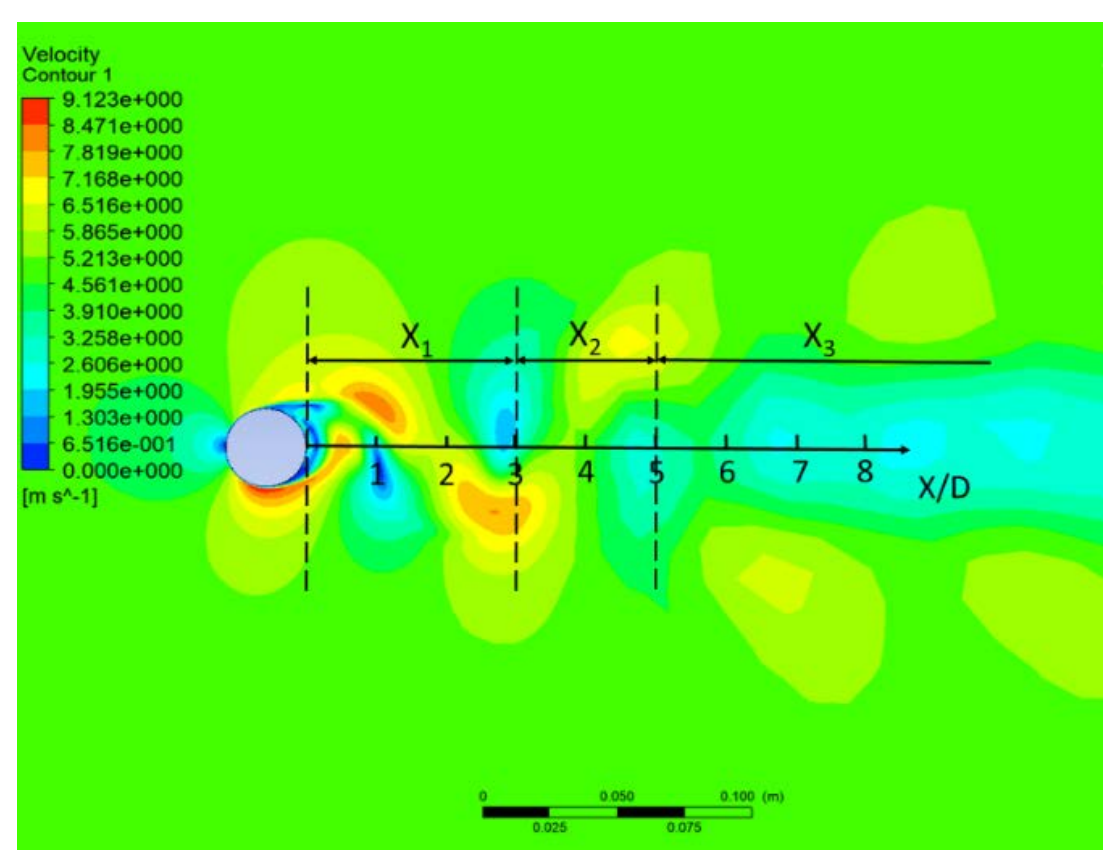

Figure 6: Instantaneous velocity contours for flow around a cylinder at $R e=10000$.

Fig. 6 shows the instantaneous wake pattern represented by velocity contours. Largescale globally synchronized vortices can be seen shedding alternately from one side of the cylinder and then the other side; these vortices are subsequently advected downstream by the underlying flow. Unal and Rockwell [52] identified three distinct regions in the wake of a cylinder: the pre-vortex formation region $\left(\mathrm{X}_{1}: X / D<3\right)$, the primary vortex formation region $\left(\mathrm{X}_{2}: 3<X / D<5\right)$, and the fully developed vortex formation region $\left(\mathrm{X}_{3}: X / D>5\right)$. In the $\mathrm{X}_{1}$ region, the boundary layer rolls up, creating initial vortices. In the $\mathrm{X}_{2}$ and $\mathrm{X}_{3}$ regions, the vortices grow to become fully developed, dominating the flow field. However, the circulation gradually decreases in the $\mathrm{X}_{3}$ region as a result of viscous dissipation.

\section{Flow around two tandem airfoils behind a single cylinder}

In this section, we consider two tandem airfoils positioned behind a single cylinder. The control parameter $X$ is the horizontal distance between the most downstream location on the surface of the cylinder and the LE of the fore airfoil, as shown in Fig. 7. 


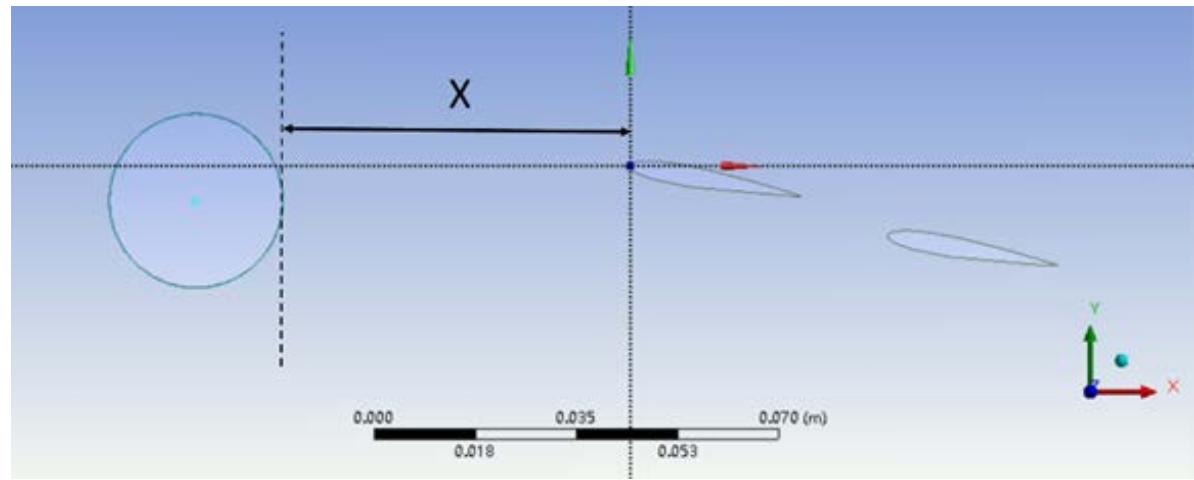

Figure 7: Geometry of two tandem airfoils behind a single cylinder.

The lift characteristics of the fore and aft airfoils at $R e=10000$ are shown in Fig. 8 and Fig. 9, respectively. Both figures contain (a) a time trace of the lift coefficient, (b) its short-time PSD, and (c) its phase portrait and Poincaré map. For each simulation run, a total sampling time of $5 \mathrm{~s}$ is used, with special care taken to allow for the passage of initial numerical transients. In Fig. 8 and Fig. 9, data spanning only $1 \mathrm{~s}$ in time are shown to highlight the temporal waveform of the lift coefficient.
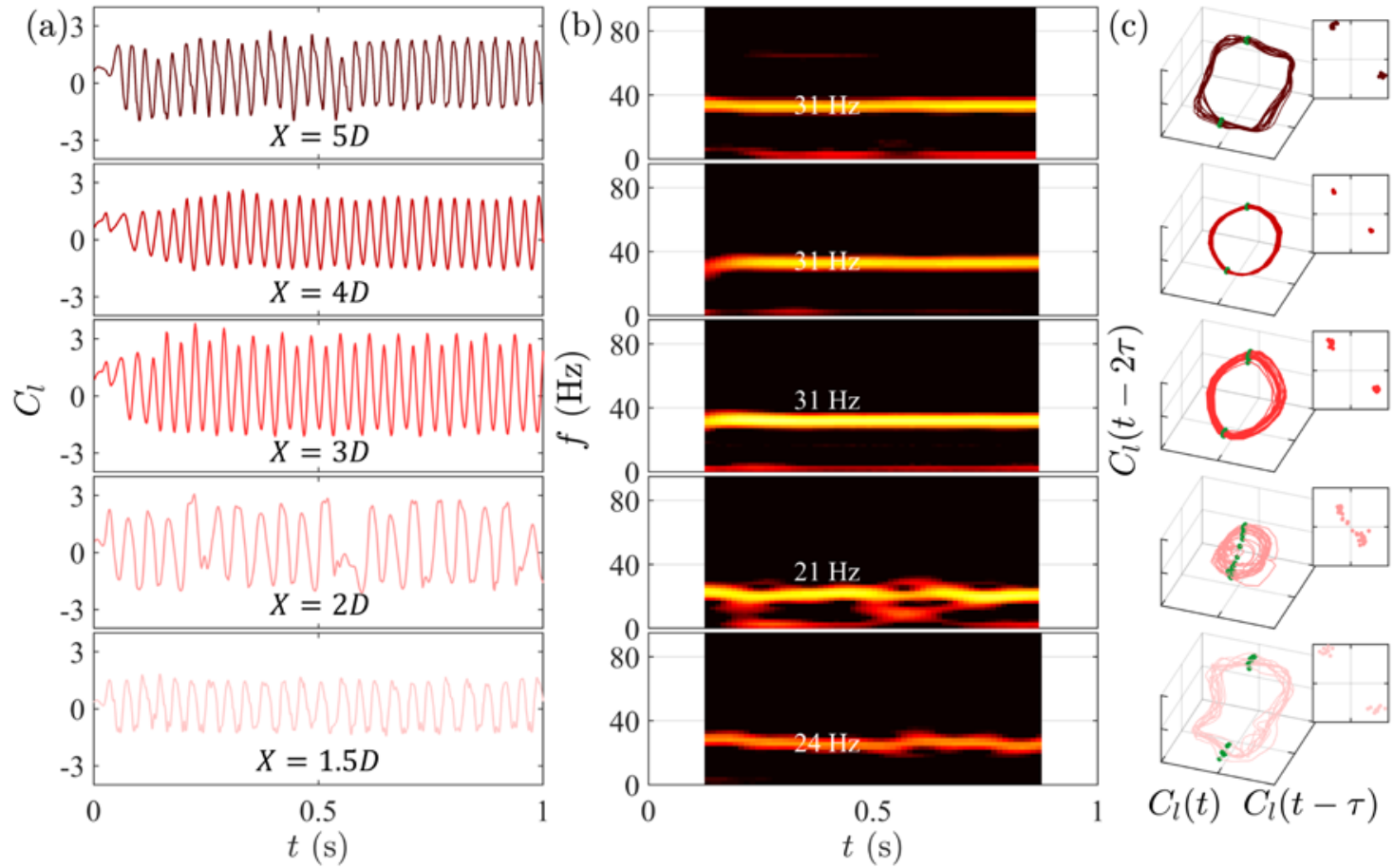

Figure 8: (a) Time trace, (b) PSD and (c) phase portrait and Poincaré map of the lift coefficient of the fore airfoil in a tandem-airfoil system behind a single cylinder at $R e$ $=10000$. The different rows correspond to different values of $X / D$. 

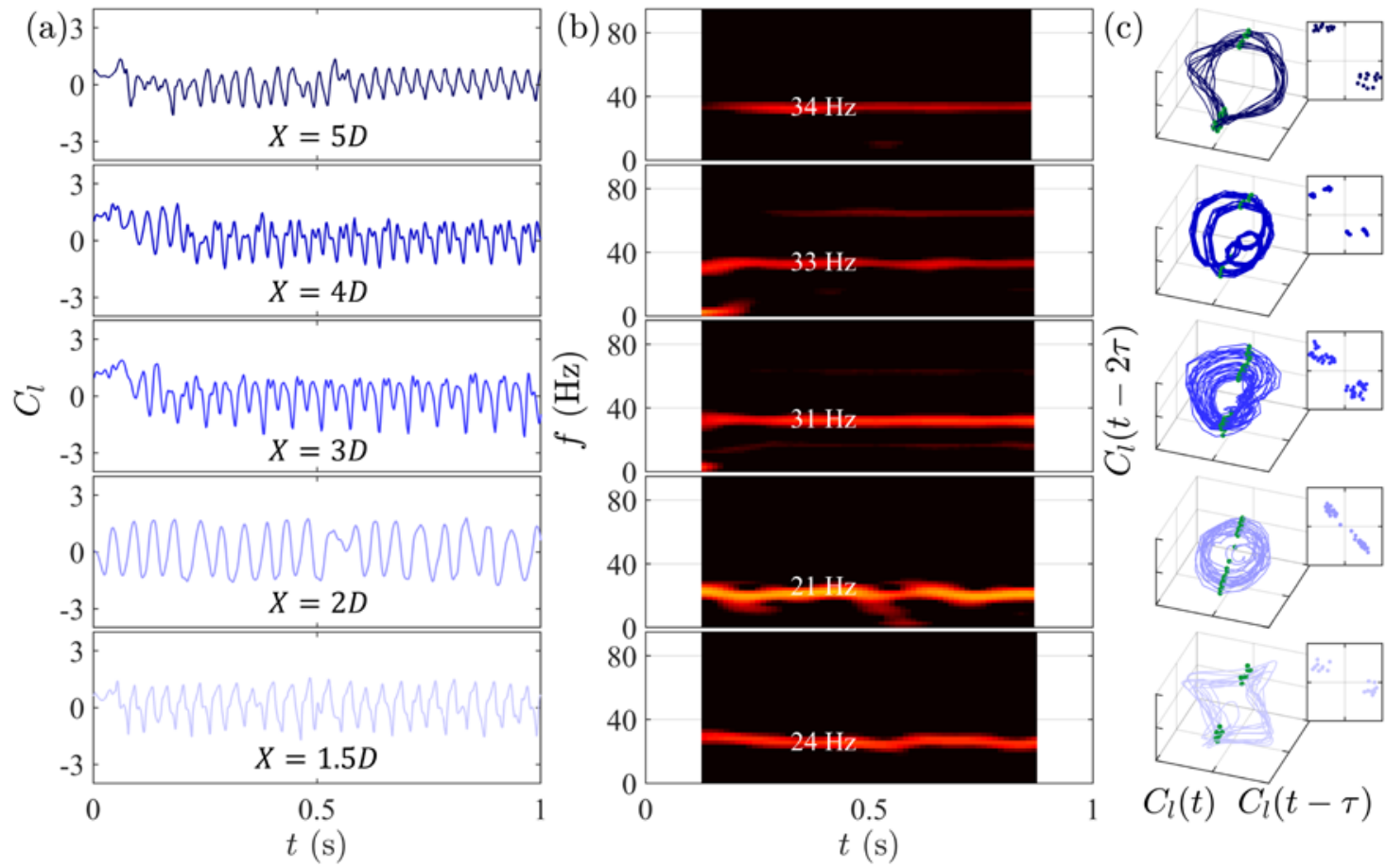

Figure 9: (a) Time trace, (b) PSD and (c) phase portrait and Poincaré map of the lift coefficient of the aft airfoil in a tandem-airfoil system behind a single cylinder at $R e=$ 10000. The different rows correspond to different values of $X / D$.

By examining Fig. 8 and Fig. 9, it can be seen that the lift forces exerted on the two tandem airfoils oscillate periodically in time. The PSD (Fig. 8(b) and Fig. 9(b)) of the lift coefficient shows a dominant peak, indicating the presence of coherent flow structures characteristic of global hydrodynamic instability [21]. Phase space reconstruction (Fig. 8(c) and Fig. 9(c)) shows a closed orbit with two discrete trajectory intercepts in the Poincaré map, indicating a period-1 limit cycle. However, under certain conditions (e.g. the aft airfoil at $X=4 D$ in Fig. 9), four discrete trajectory intercepts appear in the Poincaré map, indicating a period-2 limit cycle. Taken together, these observations are consistent with the view that these two tandem airfoils are subjected to time-periodic forcing from the global hydrodynamic mode in the self-excited cylinder wake, producing a lift force that oscillates more or less periodically at the same frequency as the global hydrodynamic mode itself.

The data from Fig. 8 and Fig. 9 are replotted in Fig. 10 to facilitate a more direct comparison across the entire range of $X / D$. It can be seen that, in terms of the Strouhal number of the dominant global mode, there is good agreement between our simulation data and the experimental data of Ozono [53], who investigated vortex shedding around a splitter plate behind a cylinder. Fig. 10(a) shows that St varies non-monotonically as $X / D$ increases, with a dip in the pre-vortex formation region (at around $X / D=2$ ) before levelling off to a value of $S t \approx 0.2$ in the primary vortex formation region $\left(\mathrm{X}_{2}: 3<X / D\right.$ $<5)$ and the fully developed vortex formation region $\left(\mathrm{X}_{3}: X / D>5\right)$. Fig. 10 (b) shows that the RMS amplitude of the lift coefficient $\left(\mathrm{Cl}_{l}\right)$ differs between the two airfoils, with the fore airfoil experiencing consistently larger variations in $C_{l}$ than the aft airfoil. This 
difference in $C_{l}$ behavior could potentially be exploited for the optimization of energy harvesting devices.
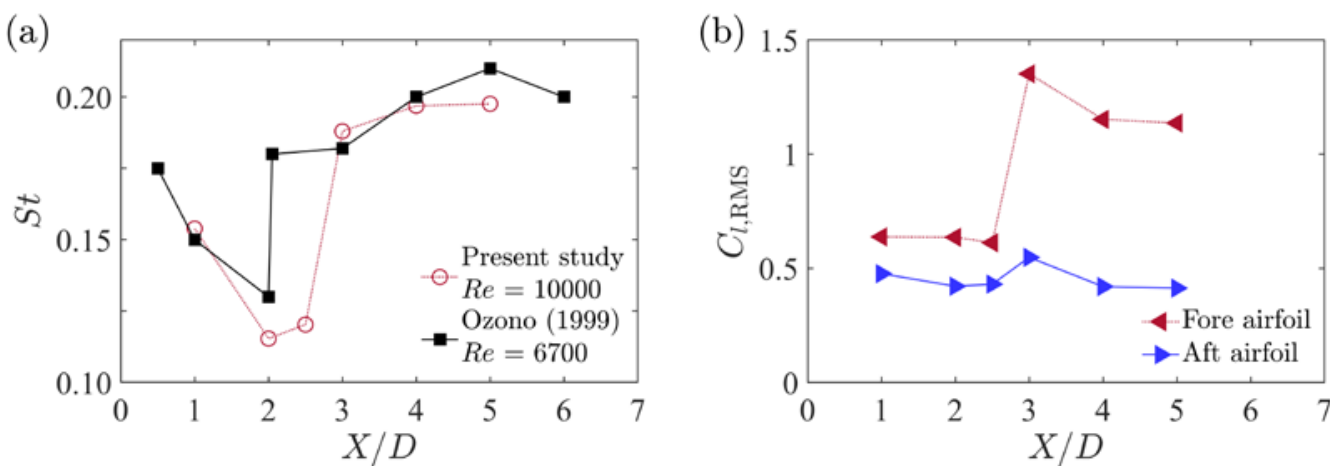

Figure 10: (a) Strouhal number of the dominant global mode and (b) RMS amplitude of the lift coefficient for two tandem airfoils behind a single cylinder at different $X / D$. Comparison data from the experiments of Ozono [53] are also shown.

Within the pre-vortex formation region $\left(\mathrm{X}_{1}: X / D<3\right)$, the shear layer from the cylinder is forced to extend downstream until it reaches the TE of the fore airfoil. At the TE, however, the shear layer rolls up, shedding vortices, which can be seen in the instantaneous velocity contours of Fig. 11(a). At these conditions, the cylinder and the fore airfoil are close enough together that they can be regarded as a consolidated "extended body".
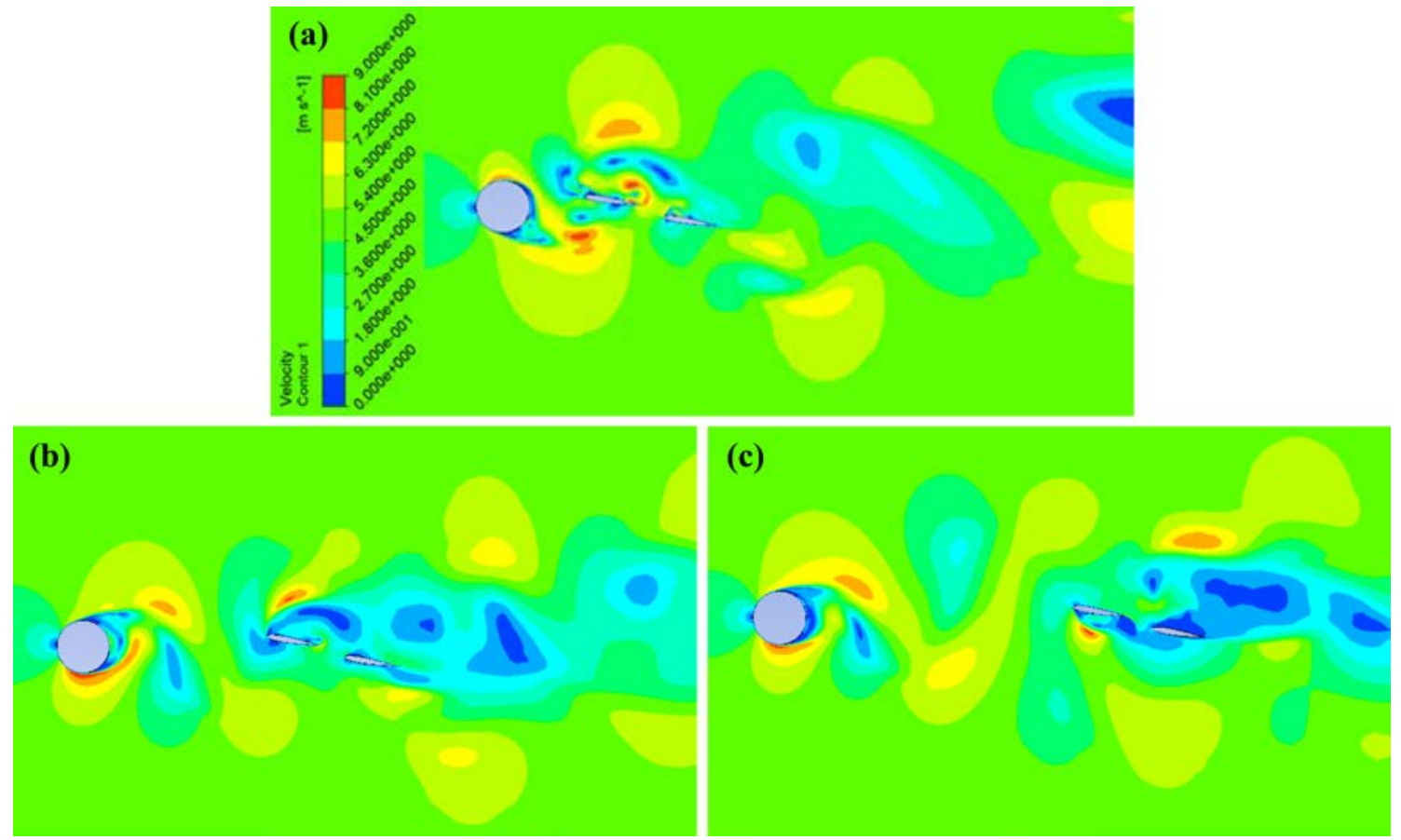

Figure 11: Instantaneous velocity contours for two tandem airfoils behind a cylinder. Data are shown for (a) the $X_{1}$ region $(X / D=1)$, (b) the $X_{2}$ region $(X / D=3)$ and (c) the $\mathrm{X}_{3}$ region $(X / D=5)$. 
On approaching the primary vortex formation region $\left(\mathrm{X}_{2}: 3<X / D<5\right)$, we observe abrupt changes in both St (Fig. 10(a)) and the RMS amplitude of $C_{l}$ (Fig. 10(b)). In particular, St jumps to around 0.2, while the RMS amplitude of $C_{l}$ of the fore airfoil jumps to around 1.3. In experiments by Ozono [53], a splitter plate was placed in a cylinder wake at $R e=6700$ and a similar jump in St was observed. This abrupt change could be explained by the termination of the "extended body" mentioned earlier. The distance between the cylinder and the fore airfoil is now long enough for vortices to form and grow. These vortices periodically impinge on the airfoils, explaining the jump in $S t$ to a classic value of 0.2 . However, it is worth noting that a similarly abrupt change in the RMS amplitude of $C_{l}$ of the aft airfoil does not occur (Fig. 10(b)) because this airfoil is farther away from the cylinder than the fore airfoil and is also shielded by it.

Within the primary vortex formation region $\left(\mathrm{X}_{2}: 3<X / D<5\right)$ and the fully developed vortex formation region $\left(\mathrm{X}_{3}: X / D>5\right)$, St remains relatively constant (at a classic value of 0.2 ) owing to the full and consistent impingement of wake vortices, as shown in the instantaneous velocity contours of Fig. 11(b) and Fig. 11(c). The RMS amplitude of $C_{l}$ of the fore airfoil reaches its maximum value and then gradually decreases as $X / D$ increases, which could be explained by the increasing role of dissipation.

\section{Flow around two tandem airfoils behind two tandem}

\section{cylinders}

In this section, we investigate the interactions between (i) the globally unstable wake of two tandem cylinders and (ii) two tandem airfoils. The horizontal distance between the center of the downstream cylinder and the LE of the fore airfoil is fixed at $X=4 D$, as shown in Fig. 12. This distance ensures sufficient horizontal spacing for vortices to form and shed from the downstream cylinder. The horizontal center-to-center spacing between the upstream and downstream cylinders $L$ is varied.

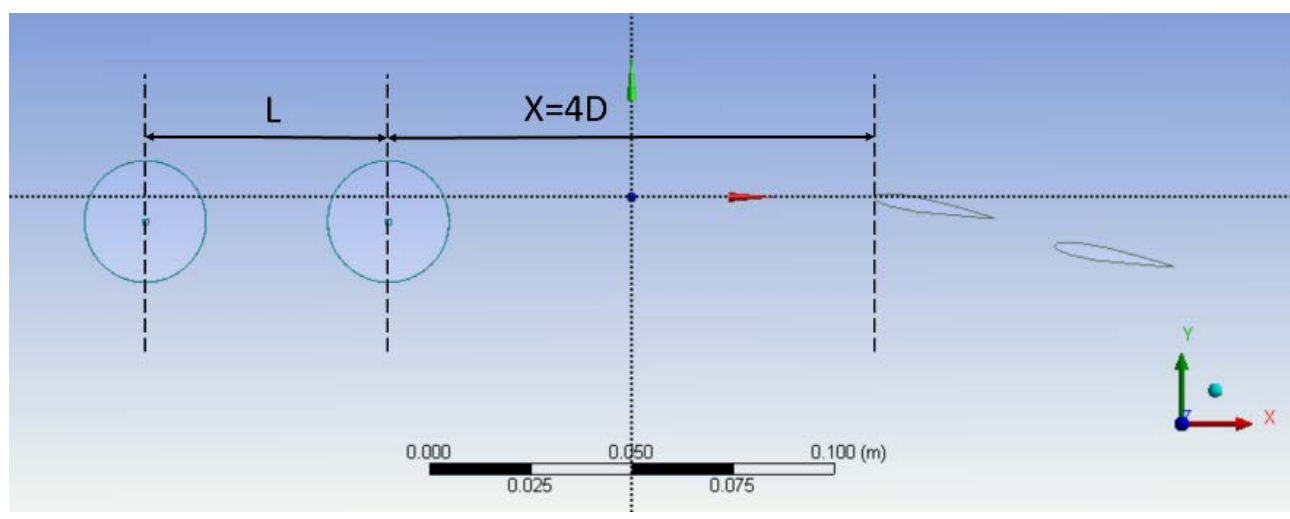

Figure 12: Geometry of two tandem airfoils behind two tandem cylinders.

As with the single-cylinder case (Section 4), the lift coefficients of the fore airfoil (Fig. 
13) and the aft airfoil (Fig. 14) are examined together at $R e=10000$. It can be seen that the oscillations in $C_{l}$ are noticeably less periodic here than they were with just a single cylinder (Section 4), as evidenced by the more irregular waveform in the time trace (Fig. 13(a) and Fig. 14(a)), the presence of weak broadband components in the PSD (Fig. 13(b) and Fig. 14(b)) and the complex structure of the attractor in phase space (Fig. 13(c) and Fig. 14(c)). At all values of $L / D$, the attractor appears to have non-closed orbits with fractal features reminiscent of chaos. Attempts were made to test for chaos using tools from dynamical systems theory, but the results were inconclusive owing to the short duration of the time traces provided by our simulations. In future work, a full analysis of such complex dynamics, using recurrence plots and other nonlinear tools, should be carried out to better understand the coupling between the self-excited cylinder wake and the tandem airfoils. Such analyses have been performed before for various flow systems, such as low-density jets [25,30,31], jet diffusion flames [33], and thermoacoustic systems [54].
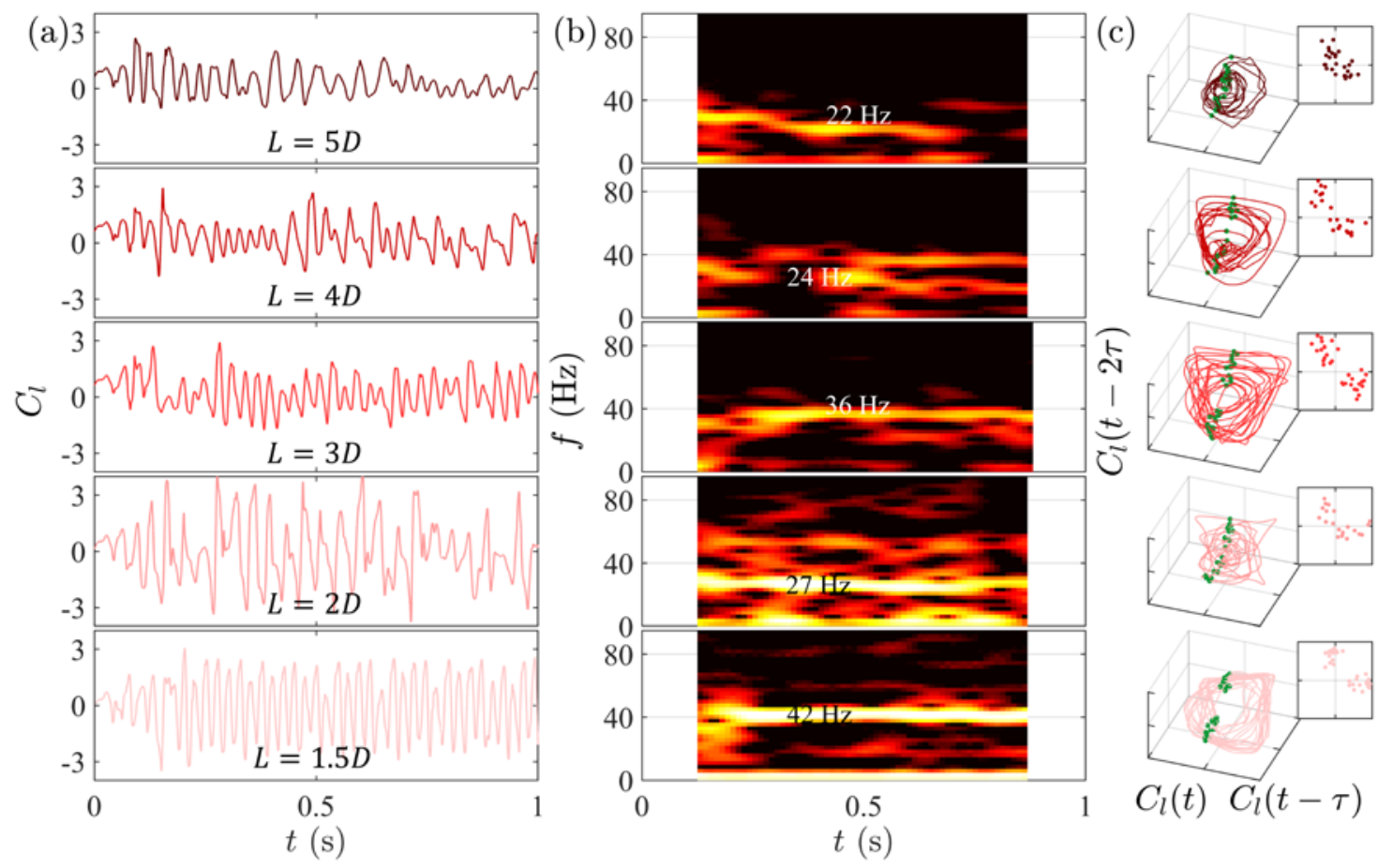

Figure 13: (a) Time trace, (b) PSD and (c) phase portrait and Poincaré map of the lift coefficient of the fore airfoil in a tandem-airfoil system behind two tandem cylinders at $R e=10000$. The different rows correspond to different values of $L / D$. 

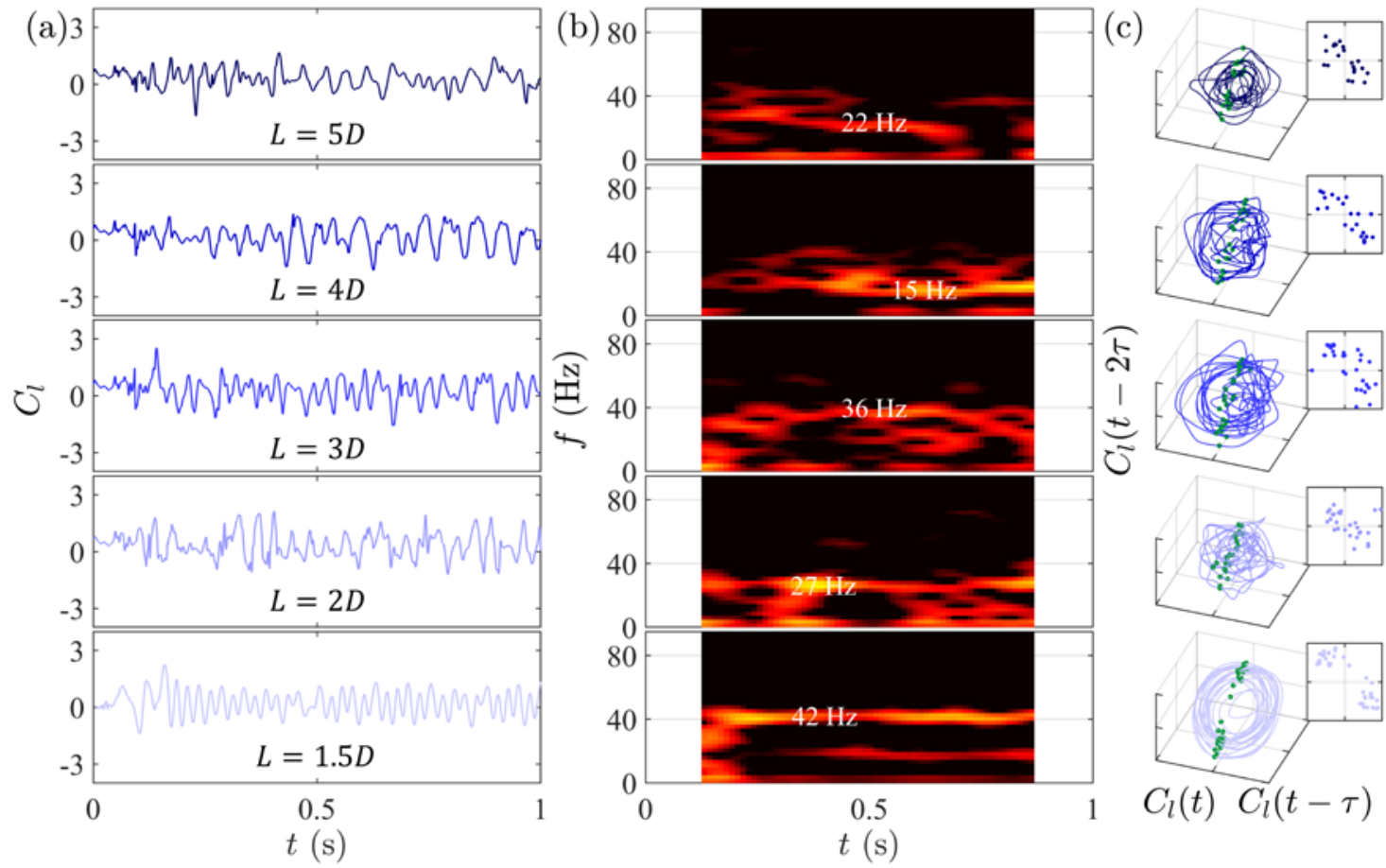

Figure 14: (a) Time trace, (b) PSD and (c) phase portrait and Poincaré map of the lift coefficient of the aft airfoil in a tandem-airfoil system behind two tandem cylinders at $R e=10000$. The different rows correspond to different values of $L / D$.

(a)

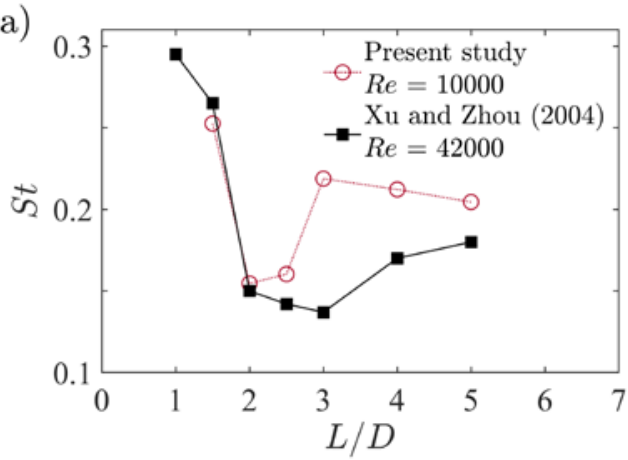

(b)

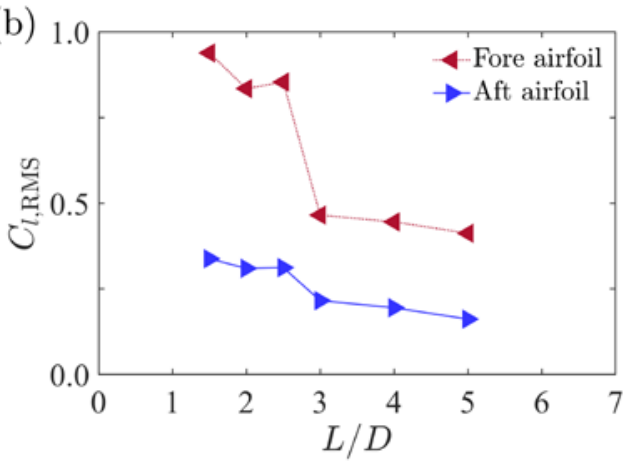

Figure 15: (a) Strouhal number of the dominant global mode and (b) RMS amplitude of the lift coefficient for two tandem airfoils behind two tandem cylinders at different $L / D$. Comparison data from the experiments of $\mathrm{Xu}$ and Zhou [55] are also shown.

Despite the irregular waveform of the time traces (Fig. 13(a) and Fig. 14(a)), it is still possible to identify, at each value of $L / D$, a dominant spectral peak in the PSD amidst weaker broadband components. Data from Fig. 13 and Fig. 14 are replotted in Fig. 15 to facilitate a more direct comparison of St and the RMS amplitude of $\mathrm{Cl}_{\text {l }}$ across the entire range of $L / D$. For St, data from the experiments of $\mathrm{Xu}$ and Zhou [55] are also shown for comparison. At small values of $L / D$ (Fig. 15(a): $1<L / D<2$ ), St is initially higher than that for the single-cylinder case $(S t=0.2)$ but it rapidly decreases as $L / D$ increases. In this specific regime, the two cylinders are so close together that they can in effect be treated as a consolidated "extended body" [56]. The flow field for this specific regime is shown in Fig. 16(a) in terms of the instantaneous velocity contours. 
It can be seen that the shear layer separating from the upstream cylinder engulfs the downstream cylinder entirely, and its length is longer than that observed in the singlecylinder case (Section 4).

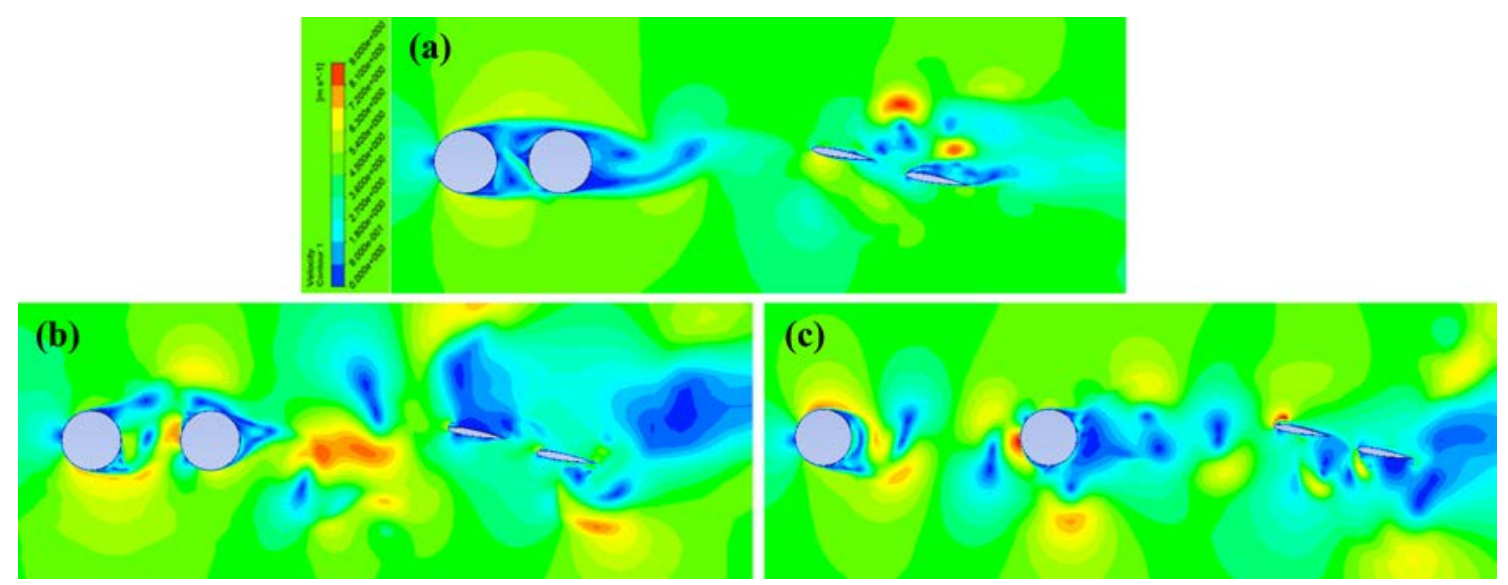

Figure 16: Instantaneous velocity contours for two tandem airfoils behind two tandem cylinders. Data are shown for (a) $L=1.5 D$, (b) $L=2.5 D$ and (c) $L=4 D$.

At intermediate values of $L / D$ (Fig. 15(a): $2<L / D<3$ ), St increases with $L / D$ until it reaches close to the classic value of $S t=0.2$. In this specific regime, the downstream cylinder is far enough from the upstream cylinder that the shear layer separating from the upstream cylinder can no longer engulf the downstream cylinder entirely. Instead, the shear layer separating from the upstream cylinder impinges on the downstream cylinder, influencing the development of the boundary layer on its surface (Fig. 16(b)). However, some degree of shear layer reattachment [57] can still be observed (Fig. 16(b)). In both the "extended body" and "reattachment" regimes, the RMS amplitude of $C_{l}$ of the fore and aft airfoils is around 0.9 and 0.3 , respectively. The ratio of these two values is close to that of the values found in the $\mathrm{X}_{2}$ and $\mathrm{X}_{3}$ regions in the singlecylinder case (Section 4 ). At around $L / D=3$, St increases sharply from around 0.15 to slightly above 0.2 (Fig. 15(a)). This increase in St is accompanied by a decrease in the RMS amplitude of $C_{l}$ of both the fore and aft airfoils - to around 50\% of their original values. This behavior is believed to be due to the termination of the reattachment regime, signalling the start of the so-called "co-shedding" regime [56].

At larger values of $L / D(L / D>3)$, the downstream cylinder is sufficiently far from the upstream cylinder that self-excited vortex shedding occurs from both cylinders at the same frequency (around $S t=0.2$ ). In this "co-shedding” regime, vortex shedding from the downstream cylinder is influenced by the impingement of vortices generated by the upstream cylinder (Fig. 16(c)). The sudden decrease in the RMS amplitude of $C_{l}$ at the inception of this regime (Fig. 15(b): $L / D=3$ ) suggests a rapid decrease in the vortex energy behind the downstream cylinder. 


\section{Flow around two tandem airfoils behind a single heated cylinder}

The onset and characteristics of global hydrodynamic instability are known to depend on the velocity and density profiles of the base flow [21]. In this section, we investigate the lift characteristics of two tandem airfoils behind a single cylinder when the cylinder is heated to a temperature above that of the free-stream. The mesh geometry and inlet flow conditions are identical to those used in Section 4, except that here the temperature of the cylinder surface is increased. The parameter $X$ is held constant at $X / D=1$. In the numerical setup, the density of air follows that of an incompressible ideal gas. The specific heat is assumed to be constant, which is a reasonable assumption given the limited range over which the temperature varies here (288 to $800 \mathrm{~K}$ ).

The lift characteristics of the fore and aft airfoils at $R e=10000$ are shown in Fig. 17 and Fig. 18, respectively, for three different cylinder temperatures ( $T=400,600,800$ $\mathrm{K})$ above the baseline temperature $(T=288 \mathrm{~K})$. Both figures contain (a) a time trace of the lift coefficient, (b) its short-time PSD, and (c) its phase portrait and Poincaré map. On examining Fig. 17 and Fig. 18, we find that increasing $T$ has little effect on the qualitative state of the system, with both the fore and aft airfoils experiencing periodic oscillations in $C$, as evidenced by the repetitive waveform of the time trace (Fig. 17(a) and Fig. 18(a)) and by the presence of a sharp dominant peak in the PSD (Fig. 17(b) and Fig. 18(b)). The trajectory of the system in phase space evolves along a closed orbit (Fig. 17(c) and Fig. 18(c)), with two blobs of intercepts in the Poincaré map, confirming the period-1 limit-cycle nature of the oscillations in $C_{l}$.

To explore the quantitative effect of $T$, we replot the data from Fig. 17 and Fig. 18 in Fig. 19 in terms of $S t$ and the RMS amplitude of $C l$. We find that increasing $T$ from the baseline value of $288 \mathrm{~K}$ to a maximum of $800 \mathrm{~K}$ leads to monotonic decreases in St as well as in the RMS amplitude of $C_{l}$ of both the fore and aft airfoils. This shows that although increasing $T$ does not change the qualitative dynamics of the system (which remains in a periodic limit cycle), it quantitatively changes the oscillation frequency and amplitude of $C_{l}$ for both the fore and aft airfoils. The decrease in the RMS amplitude of $C_{l}$ may be attributed to the airfoils encountering a lower density flow field (Fig. 20), which reduces the dynamic pressure experienced by the airfoils. 

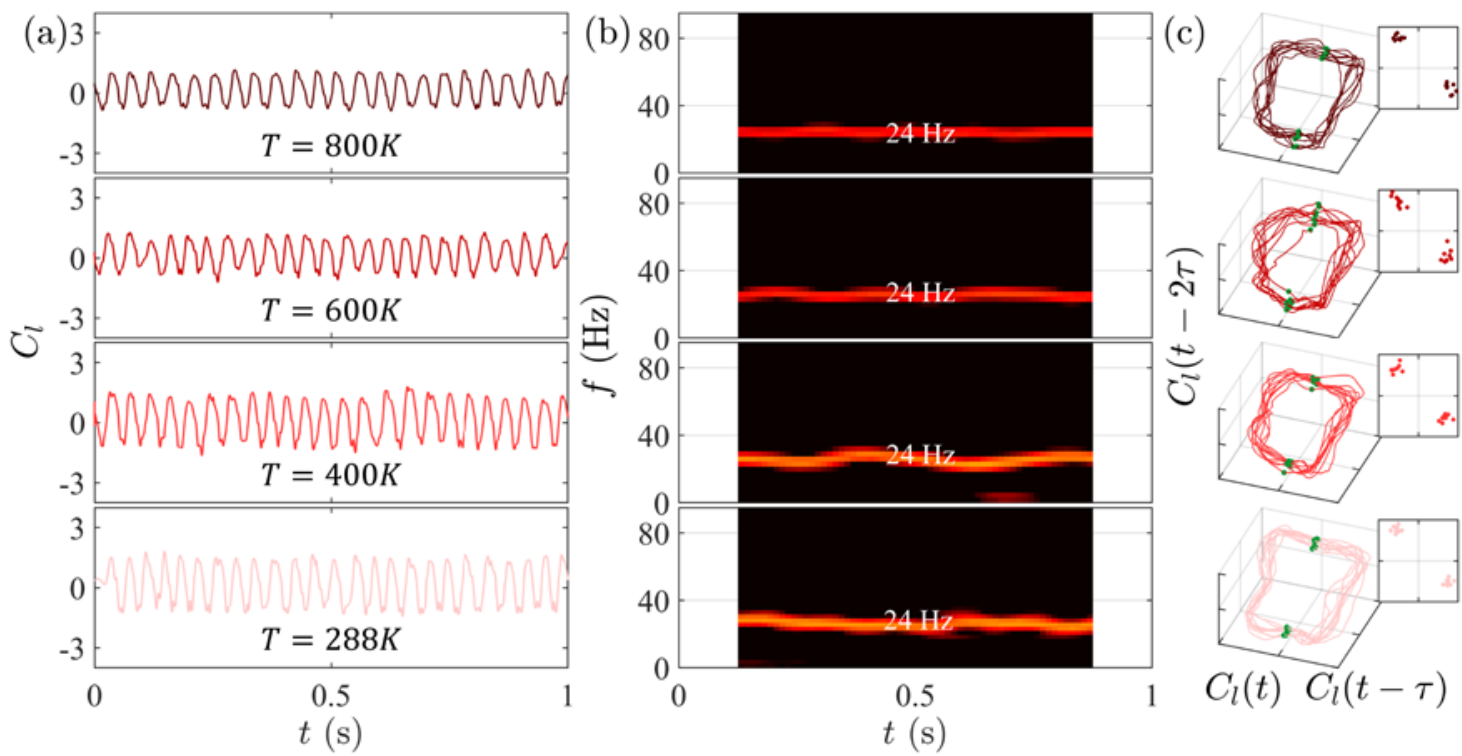

Figure 17: (a) Time trace, (b) PSD and (c) phase portrait and Poincaré map of the lift coefficient of the fore airfoil in a tandem-airfoil system behind a single heated cylinder at $R e=10000$. The different rows correspond to different values of the cylinder temperature.
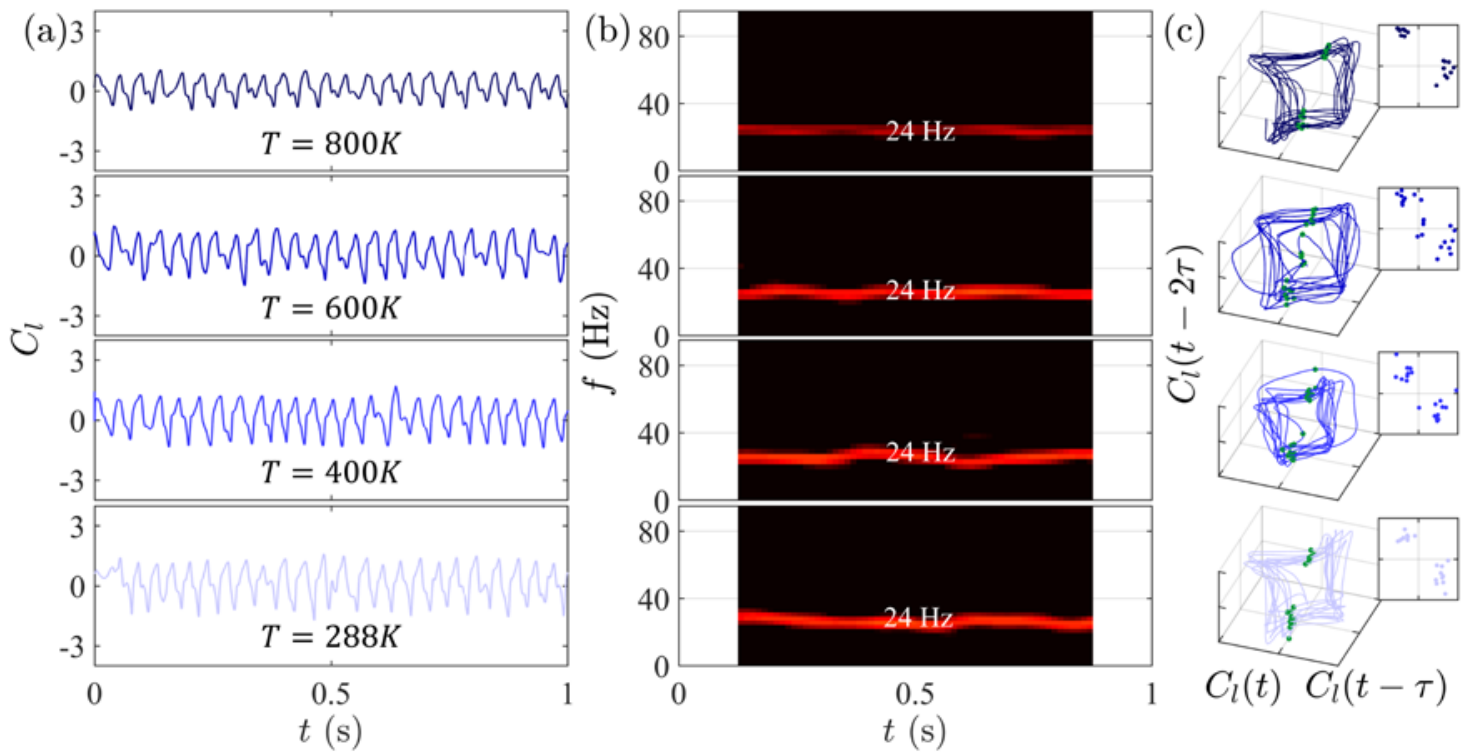

Figure 18: (a) Time trace, (b) PSD and (c) phase portrait and Poincaré map of the lift coefficient of the aft airfoil in a tandem-airfoil system behind a single heated cylinder at $R e=10000$. The different rows correspond to different values of the cylinder temperature. 

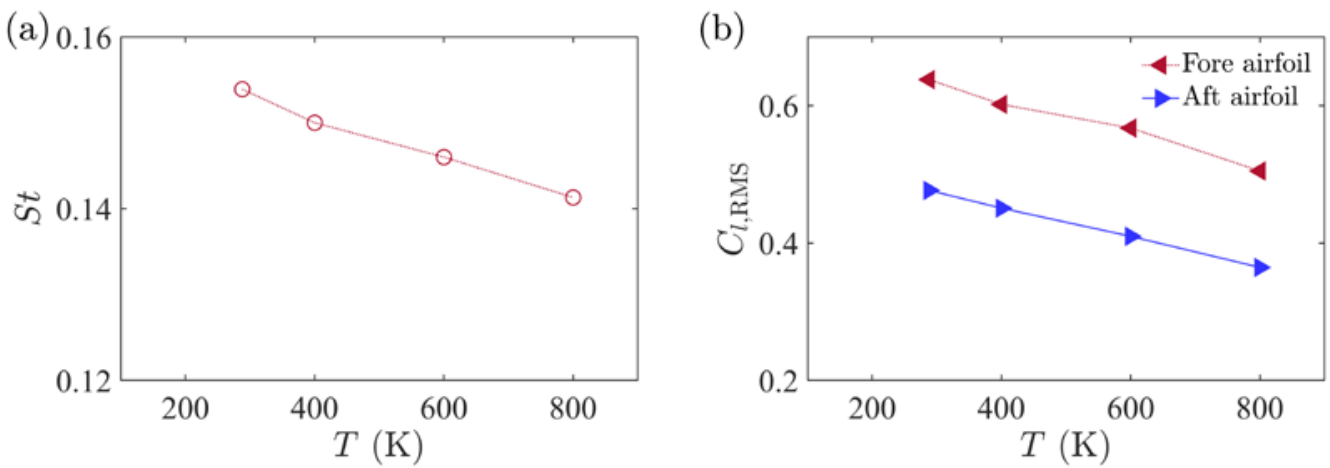

Figure 19: (a) Strouhal number of the dominant global mode and (b) RMS amplitude of the lift coefficient for two tandem airfoils behind a single cylinder heated to different temperatures.

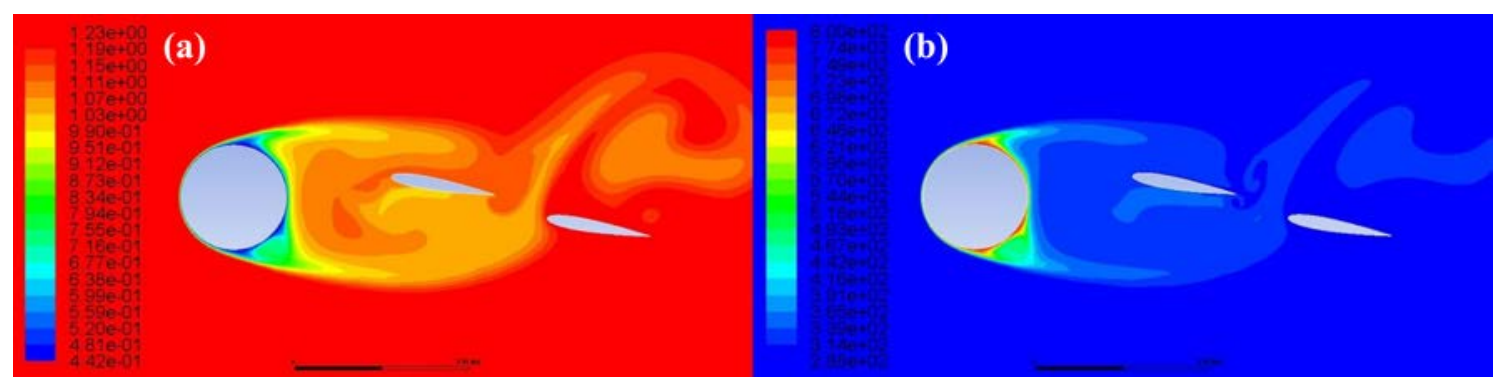

Figure 20: Instantaneous contours of (a) density and (b) temperature for two tandem airfoils behind a single cylinder heated to $T=800 \mathrm{~K}$.

\section{Conclusions}

In this study, we have numerically investigated the interactions between (i) the globally unstable self-excited wake generated by one or two rigid cylinders and (ii) two tandem airfoils positioned downstream. Two-dimensional scale-adaptive simulations were performed in the transient flow regime at $R e=10000$, with and without cylinder heating. The main findings can be summarized as follows:

1. When the two tandem airfoils are positioned behind a single cylinder (Section 4), their lift characteristics depend strongly on $X$, the horizontal spacing between the cylinder and the two airfoils. Both the fore and aft airfoils experience periodic limitcycle oscillations in $C_{l}$ at a common frequency. As $X / D$ increases from 1 to 5, St starts off at around 0.15 , drops to a minimum of 0.12 at $X / D=2$ and then jumps abruptly at $X / D=3$, eventually reaching an asymptotic value of 0.2 . Meanwhile, like the behavior of $S t$, the RMS amplitude of $C_{l}$ of the fore airfoil also jumps abruptly at $X / D=3$. This jump can be attributed to the termination of the "extended body" region observed at small values of $X / D$.

2. When the two tandem airfoils are positioned behind two tandem cylinders (Section 5), their lift coefficients oscillate less periodically than they do in the single-cylinder 
case (Section 4), with broadband components appearing in the PSD and with complex structures appearing in the attractors in phase space. Nevertheless, the quantitative lift characteristics depend strongly on $L$, the horizontal spacing between the upstream and downstream cylinders. As $L / D$ increases from 1.5 to 5 , St starts off at around 0.25, drops to a minimum of 0.15 at $L / D=2$ and then jumps abruptly at $L / D=3$, eventually reaching an asymptotic value of 0.2 . This trend in St is similar to that observed in the single-cylinder case when $X / D$ increases. However, unlike the single-cylinder case, here increasing $L / D$ through a value of 3 causes the RMS amplitude of $C_{l}$ of both the fore and aft airfoils to decrease.

3. When the two tandem airfoils are positioned behind a single cylinder heated to a temperature $T$ above that of the free-stream (Section 6), the qualitative dynamics of $\mathrm{Cl}_{l}$ is similar to that observed in the isothermal case (Section 4), with $\mathrm{Cl}_{l}$ of both airfoils oscillating periodically in a limit cycle. However, increasing $T$ from $288 \mathrm{~K}$ (baseline) to $800 \mathrm{~K}$ leads to monotonic decreases in St as well as in the RMS amplitude of $C_{l}$ of both airfoils.

\section{References}

[1] Maleki, A., Elahi, M., Assad, M.E.H., Nazari, M.A., Shadloo, M.S. and Nabipour, N., 2020. Thermal conductivity modeling of nanofluids with ZnO particles by using approaches based on artificial neural network and MARS. Journal of Thermal Analysis and Calorimetry, pp.1-12.

[2] Moravej, M., Saffarian, M.R., Li, L.K.B., Doranehgard, M.H. and Xiong, Q., 2019. Experimental investigation of circular flat-panel collector performance with spiral pipes. Journal of Thermal Analysis and Calorimetry, pp.1-8.

[3] Rashidi, S., Karimi, N., Mahian, O. and Esfahani, J.A., 2019. A concise review on the role of nanoparticles upon the productivity of solar desalination systems. Journal of Thermal Analysis and Calorimetry, 135(2), pp.1145-1159.

[4] Komeilibirjandi, A., Raffiee, A.H., Maleki, A., Nazari, M.A. and Shadloo, M.S., 2020. Thermal conductivity prediction of nanofluids containing $\mathrm{CuO}$ nanoparticles by using correlation and artificial neural network. Journal of Thermal Analysis and Calorimetry, 139(4), pp.2679-2689.

[5] Akbarzadeh, M., Rashidi, S., Karimi, N. and Omar, N., 2019. First and second laws of thermodynamics analysis of nanofluid flow inside a heat exchanger duct with wavy walls and a porous insert. Journal of Thermal Analysis and Calorimetry, 135(1), pp.177-194.

[6] Xiong, Q., Bozorg, M.V., Doranehgard, M.H., Hong, K. and Lorenzini, G., 2020. 
A CFD investigation of the effect of non-Newtonian behavior of $\mathrm{Cu}$-water nanofluids on their heat transfer and flow friction characteristics. Journal of Thermal Analysis and Calorimetry, 139(4), pp.2601-2621.

[7] Siavashi, M., Karimi, K., Xiong, Q. and Doranehgard, M.H., 2019. Numerical analysis of mixed convection of two-phase non-Newtonian nanofluid flow inside a partially porous square enclosure with a rotating cylinder. Journal of Thermal Analysis and Calorimetry, 137(1), pp.267-287.

[8] Amini, Y., Akhavan, S. and Izadpanah, E., 2019. Vortex-induced vibration of a cylinder in pulsating nanofluid flow. Journal of Thermal Analysis and Calorimetry, pp.1-16.

[9] McKinney, W., DeLaurier J., The wing mill: an oscillating-wing windmill, Journal of Energy 5 (2) (1981) 109-115.

[10] Lau, Y.L., So, R.M.C., Leung, R.C.K., Flow-induced vibration of elastic slender structures in a cylinder wake, Journal of Fluids and Structures, 2004

[11] Derakhshandeh, J.F., Arjomandi, M., Dally, B., Cazzolato, B., Flow-induced vibration of an elastically mounted airfoil under the influence of the wake of a circular cylinder, Experimental Thermal and Fluid Science 74 (2016) 58-72

[12] He, W., Yu, P. and Li, L.K.B., 2018. Ground effects on the stability of separated flow around a NACA 4415 airfoil at low Reynolds numbers. Aerospace Science and Technology, 72, pp.63-76.

[13] He, W., Guan, Y., Theofilis, V. and Li, L.K.B., 2019. Stability of low-Reynoldsnumber separated flow around an airfoil near a wavy ground. AIAA Journal, 57(1), pp.29-34.

[14] He, W., Pérez, J.M., Yu, P. and Li, L.K.B., 2019. Non-modal stability analysis of low-Re separated flow around a NACA 4415 airfoil in ground effect. Aerospace Science and Technology, 92, pp.269-279.

[15] Kurtulus, D.F., 2016. On the wake pattern of symmetric airfoils for different incidence angles at $\mathrm{Re}=1000$. International Journal of Micro Air Vehicles, 8(2), pp.109-139.

[16] Kurtulus, D.F., 2015. On the unsteady behavior of the flow around NACA 0012 airfoil with steady external conditions at $\mathrm{Re}=1000$. International Journal of Micro Air Vehicles, 7(3), pp.301-326.

[17] Kurtulus, D.F., 2018. Aerodynamic loads of small-amplitude pitching NACA 0012 
airfoil at Reynolds number of 1000. AIAA Journal, 56(8), pp.3328-3331.

[18] Rossi, E., Colagrossi, A., Oger, G. and Le Touzé, D., 2018. Multiple bifurcations of the flow over stalled airfoils when changing the Reynolds number. Journal of Fluid Mechanics, 846, pp.356-391.

[19] Durante, D., Rossi, E. and Colagrossi, A., 2020. Bifurcations and chaos transition of the flow over an airfoil at low Reynolds number varying the angle of attack. Communications in Nonlinear Science and Numerical Simulation, 89, p.105285.

[20] Yin, B., Guan, Y., Wen, A., Karimi, N. and Doranehgard, M.H., 2020. Numerical simulations of ultra-low-Re flow around two tandem airfoils in ground effect: isothermal and heated conditions. Journal of Thermal Analysis and Calorimetry, pp.117.

[21] Huerre, P. and Monkewitz, P.A., 1990. Local and global instabilities in spatially developing flows. Annual Review of Fluid Mechanics, 22(1), pp.473-537.

[22] Provansal, M., Mathis, C. and Boyer, L., 1987. Bénard-von Kármán instability: transient and forced regimes. Journal of Fluid Mechanics, 182, pp.1-22.

[23] Zhu, Y., Gupta, V. and Li, L.K.B., 2019. Coherence resonance in low-density jets. Journal of Fluid Mechanics, 881, R1.

[24] Kyle, D.M. and Sreenivasan, K.R., 1993. The instability and breakdown of a round variable-density jet. Journal of Fluid Mechanics, 249, pp.619-664.

[25] Murugesan, M., Zhu, Y. and Li, L.K.B., 2019. Complex network analysis of forced synchronization in a hydrodynamically self-excited jet. International Journal of Heat and Fluid Flow, 76, pp.14-25.

[26] Lee, M., Zhu, Y., Li, L.K.B. and Gupta, V., 2019. System identification of a lowdensity jet via its noise-induced dynamics. Journal of Fluid Mechanics, 862, pp.200215.

[27] Monkewitz, P.A., Bechert, D.W., Barsikow, B. and Lehmann, B., 1990. Selfexcited oscillations and mixing in a heated round jet. Journal of Fluid Mechanics, 213, pp.611-639.

[28] Raghu, S. and Monkewitz, P.A., 1991. The bifurcation of a hot round jet to limitcycle oscillations. Physics of Fluids A: Fluid Dynamics, 3(4), pp.501-503.

[29] Zhu, Y., Gupta, V. and Li, L.K.B., 2017. Onset of global instability in low-density jets. Journal of Fluid Mechanics, 828, R1. 
[30] Li, L.K.B. and Juniper, M.P., 2013. Phase trapping and slipping in a forced hydrodynamically self-excited jet. Journal of Fluid Mechanics, 735, R5.

[31] Li, L.K.B. and Juniper, M.P., 2013. Lock-in and quasiperiodicity in a forced hydrodynamically self-excited jet. Journal of Fluid Mechanics, 726, pp.624-655.

[32] Juniper, M.P., Li, L.K.B. and Nichols, J.W., 2009. Forcing of self-excited round jet diffusion flames. Proceedings of the Combustion Institute, 32(1), pp.1191-1198.

[33] Li, L.K.B. and Juniper, M.P., 2013. Lock-in and quasiperiodicity in hydrodynamically self-excited flames: Experiments and modelling. Proceedings of the Combustion Institute, 34(1), pp.947-954.

[34] ANSYS Fluent User's Guide, ANSYS, Inc. Release 17.0, Southpointe November 2013, 275 Technology Drive, Canonsburg, PA 15317

[35] Maliska, C.R., Paladino E.E., Saltara F., Contessi B.A., Ataides R., Girardi Silva V., A comparison of turbulence models for the computation of a detached flow around a square cylinder, 20th Annual Conference of the CFD Society of Canada, Computational Fluid Dynamic Society of Canada, Paper S5A03, Canada, 9-11 May 2012.

[36] Mentor, F.R., ANSYS Germany GmbH, 2012. Best Practice: Scale-Resolving Simulations in ANSYS CFD (Version 1.0)

[37] Kabiraj, L. and Sujith, R.I., 2012. Nonlinear self-excited thermoacoustic oscillations: intermittency and flame blowout. Journal of Fluid Mechanics, 713, pp. 376-397.

[38] Kabiraj, L., Sujith, R.I. and Wahi, P., 2012. Bifurcations of self-excited ducted laminar premixed flames. Journal of Engineering for Gas Turbines and Power, 134(3), p.031502.

[39] Kabiraj, L., Saurabh, A., Wahi, P. and Sujith, R.I., 2012. Route to chaos for combustion instability in ducted laminar premixed flames. Chaos: An Interdisciplinary Journal of Nonlinear Science, 22(2), p.023129.

[40] Hachijo, T., Masuda, S., Kurosaka, T. and Gotoda, H., 2019. Early detection of thermoacoustic combustion oscillations using a methodology combining statistical complexity and machine learning. Chaos: An Interdisciplinary Journal of Nonlinear Science, 29(10), p.103123.

[41] Aoki, C., Gotoda, H., Yoshida, S. and Tachibana, S., 2020. Dynamic behavior of 
intermittent combustion oscillations in a model rocket engine combustor. Journal of Applied Physics, 127(22), p.224903.

[42] Gotoda, H., Shinoda, Y., Kobayashi, M., Okuno, Y. and Tachibana, S., 2014. Detection and control of combustion instability based on the concept of dynamical system theory. Physical Review E, 89(2), p.022910.

[43] Balusamy, S., Li, L.K.B., Han, Z. and Hochgreb, S., 2017. Extracting flame describing functions in the presence of self-excited thermoacoustic oscillations. Proceedings of the Combustion Institute, 36(3), pp.3851-3861.

[44] Guan, Y., Gupta, V., Wan, M. and Li, L.K.B., 2019. Forced synchronization of quasiperiodic oscillations in a thermoacoustic system. Journal of Fluid Mechanics, 879, pp.390-421.

[45] Rigas, G., Jamieson, N.P., Li, L.K.B. and Juniper, M.P., 2016. Experimental sensitivity analysis and control of thermoacoustic systems. Journal of Fluid Mechanics, 787.

[46] Kashinath, K., Li, L.K.B. and Juniper, M.P., 2018. Forced synchronization of periodic and aperiodic thermoacoustic oscillations: lock-in, bifurcations and open-loop control. Journal of Fluid Mechanics, 838, pp.690-714.

[47] Guan, Y., Murugesan, M. and Li, L.K.B., 2018. Strange nonchaotic and chaotic attractors in a self-excited thermoacoustic oscillator subjected to external periodic forcing. Chaos: An Interdisciplinary Journal of Nonlinear Science, 28(9), p.093109.

[48] Guan, Y., Gupta, V., Kashinath, K. and Li, L.K.B., 2019. Open-loop control of periodic thermoacoustic oscillations: experiments and low-order modelling in a synchronization framework. Proceedings of the Combustion Institute, 37(4), pp.53155323.

[49] Lee, M., Guan, Y., Gupta, V. and Li, L.K.B., 2020. Input-output system identification of a thermoacoustic oscillator near a Hopf bifurcation using only fixedpoint data. Physical Review E, 101(1), p.013102.

[50] Dong, S. and Karniadakis, G.E., 2005. DNS of flow past a stationary and oscillating cylinder at $\mathrm{Re}=10000$. Journal of Fluids and Structures, 20(4), pp.519-531.

[51] Gopalkrishnan, R., 1993. Vortex-induced forces on oscillating bluff cylinders (No. WHOI-92-38). Woods Hole Oceanographic Institute.

[52] Unal, M., Rockwell, D., On vortex formation from a cylinder, Part 2, Control by splitter-plate interference, J. Fluid Mech. 190 (1988) 513-529. 
[53] Ozono, S., Flow control of vortex shedding by a short splitter plate asymmetrically arranged downstream of a cylinder, Phys. Fluids 11 (1999) 2928-2934.

[54] Guan, Y., Gupta, V. and Li, L.K.B., 2020. Intermittency route to self-excited chaotic thermoacoustic oscillations. Journal of Fluid Mechanics, 894, R3.

[55] Xu, G. and Zhou, Y., 2004. Strouhal numbers in the wake of two inline cylinders. Experiments in Fluids, 37(2), pp.248-256.

[56] Zdravkovich, M.M., 1987. The effects of interference between circular cylinders in cross flow. Journal of Fluids and Structures 1, 239-261.

[57] Zhou, Y., Yiu, M.W., 2006. Flow structure, momentum and heat transport in a two-tandem-cylinder wake. Journal of Fluid Mechanics 548, 17-48. 